\title{
App More Life. Tecnología aplicada a la disminución de violencia hacia la mujer.
}

Doctorante Agustin Cortes Coss agustin.cortesc@uanl.mx Orcid: 0000-0002-0820-6463 Universidad Autónoma de Nuevo León

Dra. Dina Elizabeth Cortes Coss elycoss@gmail.com

Orcid: 0000-0003-4150-1541 Universidad Autónoma de Nuevo León

De acuerdo a estadísticas analizadas de los años 2017, 2018 y los meses transcurridos del 2019, se ha podido observar que los índices de violencia hacia la mujer han incrementado exponencialmente en México.

Simultáneamente, la aparición de las TICs (Tecnologías de la Información y la Comunicación), han permitido una vinculación prioritaria en términos de responsabilidad social. Así, las redes que ofrecen las TICS consienten que temas como el de la violencia hacia la mujer pueda ampliar su trama hacia la detección, acción, protección de las víctimas y, sobre todo, de prevención. Cuando lo anterior se conjuga, los problemas sociales atendidos tienen otra alternativa; además de que sirven de instrumento para llegar a la ciudadanía y lograr crear una red de participación contra el maltrato machista.

Bajo el precepto anterior, y siguiendo una metodología secuencial o de cascada, se ha propuesto contribuir con el desarrollo de una App móvil denominada "more life", que emita el envío de una alerta de emergencia en caso de ser requerida; y cuyo objetivo esencial es el de contribuir a disminuir los índices de violencia además de trabajar en la prevención de esta.

Palabras clave: App móvil, violencia doméstica, mujer, México.

\section{Abstract}

According to statistics analyzed for the years 2017, 2018 and the recently months in 2019, can be observed that the rates of violence against women have increased exponentially in Mexico.

Simultaneously, the emergence of ICTs (Information and Communication Technologies) has allowed a priority link in terms of social responsibility. Thus, the networks offered by ICTs allow topics such as violence against women to broaden their scope towards detection, action, protection of victims and, above all, prevention. When the above is combined, the social problems addressed have another alternative; besides that they serve as an instrument to reach citizenship and to create a network of participation against male mistreatment.

Under the previous precept, and following a sequential or cascade methodology, it has been proposed to contribute to the development of a mobile App called "more life", which issues the sending of an emergency alert if required; and whose essential objective is to contribute to reducing the rates of violence in addition to working to prevent it.

Key words: Mobile app, domestic violence, women, Mexico.

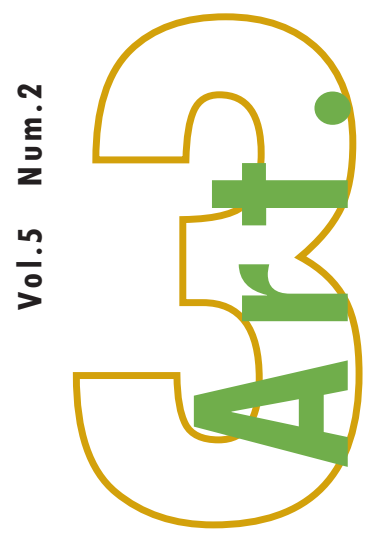




\section{DESCRIPCIÓN DEL PROBLEMA}

En Nuevo León han aparecido nuevas modalidades de delincuencia , como el robo a negocios pequeños a mano armada, los asaltos en el transporte público y últimamente los robos a instituciones universitarias así como a los transeúntes que circulan entre los espacios del campus universitario, de éste sentido se desprenden algunos actos delictivos realizados hacia mujeres que afırmaron haber sido víctimas de robos a mano armada dentro de las instalaciones de la institución; sin desmeritar a los demás grupos quienes integran la sociedad universitaria , los noticieros muestran más actos violentos emitidos hacia el sexo femenino, por tal motivo, se pretende contribuir con esta problemática mediante un sistema tecnológico que además de brindar información sobre medidas de seguridad, brinde asistencia en caso de sufrir un atentado como el descrito con anterioridad.

\section{OBJETIVOS}

Crear una aplicación móvil que contribuya a reducir la violencia que las mujeres universitarias experimentan dentro del campus. Los objetivos específicos que persigue esta investigación son:

1.Compilar información teórica dentro de la App con la finalidad de que la misma se encuentre siempre vigente para su consulta 2.Realizar un trabajo colaborativo entre instituciones para contribuir a la disminución de los índices de violencia hacia las mujeres.

\section{BASES TEÓRICAS}

De acuerdo al Instituto Nacional de Estadística y Geografía- INEGI $(2017$, p.8) se estima que tan solo en América Latina y el Caribe, $48 \%$ de los países cuenta con información sobre violencia contra las mujeres, y México aparece como uno de los más avanzados de la región en la materia.

De acuerdo a los resultados de la Encuesta Nacional sobre la Dinámica de las Relaciones en los Hogares ENDIREH (2016, p.1) en México el 43.2 por ciento de las mujeres de 15 años y más sufrió algún incidente de violencia por parte de su pareja (esposo o pareja, exesposo o expareja, o novio) durante su última relación. El porcentaje fluctúa entre 54.1 por ciento en el Estado de México y 33.7 por ciento en Coahuila de Zaragoza.

De estas mujeres, 37.5 por ciento declaró haber recibido agresiones emocionales que afectaron su salud mental y psicológica; 23.4 por ciento recibió algún tipo de agresión para controlar sus ingresos y el flujo de los recursos monetarios del hogar, así como cuestionamientos con respecto a la forma en que gastaba dicho ingreso. Afırma inMujeres (2009, pág. 4)

En un sentido más específico de la presente investigación, la aplicación que se presenta es una forma discreta para teléfonos Android que manda alertas a través de SMS y geo localizador a contactos de confianza agregados de la libreta de direcciones y a los cuales se les notificará para cualquier tipificación de violencia.

para la sustentación teórica se cuenta con documentos de investigación sobre estadísticas en violencia hacia la mujer, sin embargo, en cuestiones tecnológicas no se contaba con antecedentes, se codifico more life sin referencia alguna, simplemente tomando en cuenta casos de violencia a mujeres dentro de campos universitarios por todo el país. 


\section{DESARROLLO}

Se comenzó con el desarrollo de una manera un poco simple, el principal objetivo era el de mandar los mensajes a la persona que estaría solicitando ayuda hacia el usuario con un botón al que se le llamó "Enviar mensajes", este consistía en pedir permisos al mismo dispositivo para que el usuario le diera acceso de mandar mensajes a la aplicación y con ese mismo permiso pedir acce so a la ubicación exacta de la víctima que esté utilizando esta aplicación.

Cabe mencionar que tanto los mensajes de texto, los números de contacto al cual el usuario mandaría la alerta, un botón de "Falsa alarma" en caso de que el mensaje se haya mandado de manera accidental y la posición GPS requería permisos, pero al haber encontrado la manera de acceder a los permisos del dispositivo al mandar los mensajes, el resto de la aplicación fue desempeñado bajo la misma lógica. Se pensó en otra forma de mandar el mensaje de manera más discreta, el cual consistía en presionar 2 veces el botón de bloqueo del dispositivo para que pudiese mandar el mensaje de manera más directa y con un texto ya predeterminado, pero se encontró con un problema el cual el botón que se estaba utilizando para mandar el mensaje discreto no era virtual sino que era físico, lo cual eso significaba otro tipo de comando en la lógica del código para que pudiese ser usado de manera correcta, se comenzaron con pruebas para ver en donde se necesitaría colocar el código para que se pudiese hacer lo requerido. Después de varios intentos se solucionó el detalle de mandar los mensajes simplemente agregando un comando diferente al código el cual permite interactuar con los botones como lo son el de volumen, el de la cámara (En algunos dispositivos) y el de bloqueo y al comprobarlo ya se envían los mensajes de manera satisfactoria.

\section{METODOLOGÍA EMPLEADA}

De acuerdo a Larman C. y Basilli V. (2003, p. 47) La metodología en desarrollo en cascada, también llamado secuencial o ciclo de vida de un programa (denominado así por la posición de las fases en el desarrollo de esta, que parecen caer en cascada "por gravedad" hacia las siguientes fases), es el enfoque metodológico que ordena rigurosamente las etapas del proceso para el desarrollo de software, de tal forma que el inicio de cada etapa debe esperar a la finalización de la etapa anterior. Al final de cada etapa, el modelo está diseñado para llevar a cabo una revisión final, que se encarga de determinar si el proyecto está listo para avanzar a la siguiente fase.

Este modelo fue el primero en originarse y es la base de todos los demás modelos de ciclo de vida.

Estas fases utilizadas son:

1.Análisis de requisitos. 2.Diseño del sistema. 3.Diseño del programa. 4.Codificación. 5.Pruebas.

\section{Análisis de Requisitos}

Para los requisitos se realizaron investigaciones sobre las estadísticas de violencia hacia la mujer en México, así como la búsqueda de aplicaciones que tuvieran el mismo enfoque.

Diseño del sistema

Se acordó la realización del sistema en la plataforma Android, al ser una de las plataformas con más usuarios registrados en el mercado. EL PAIS $(2017$, p.1).

\section{Diseño del programa}

Comenzando a trabajar con lógica del programa, la función de los botones y las maneras mas viables de brindarles seguridad a las mujeres.

El diseño comenzó con colores básicos mientras la lógica se establecía, una vez terminada la lógica se comenzó a trabajar en el diseño de colores, botones y logos.

\section{Codificación}

El inicio de codificación de la aplicación se dio de manera fluida al ya tener establecido la lógica que el programa realizaría, algunos de los inconvenientes que surgieron durante la codificación fue el uso requerido de permisos que el sistema necesitaba pedir para enviar tanto los mensajes como la localización.

\section{Pruebas}

Las pruebas consistían en demostrar la funcionalidad de los botones, el uso adecuado de la información que el usuario proporcionaba, que el sistema aceptase sin ningún problema cualquier número telefónico de México y la correcta visualización de la información de centros de apoyo para las mujeres violentadas. 


\section{RESULTADOS}

Una vez comprobada la correcta funcionalidad de la aplicación, se comenzó a distribuir a teléfonos móviles.

A continuación, se muestran las pantallas resultantes de la codificación:

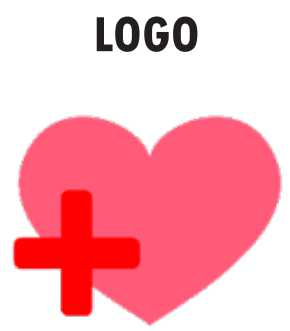

PANTALLA DE INICIO

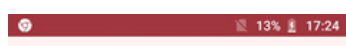

MORE LIFE

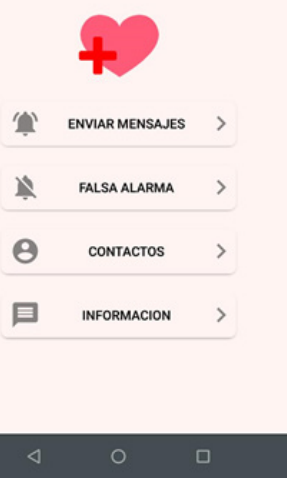

PANTALLA DE CONTACTOS

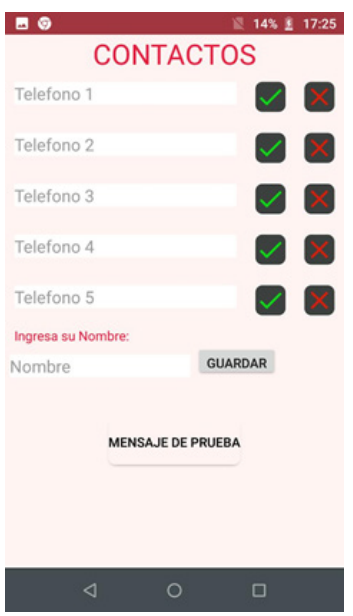

\section{PANTALLAS DE INFORMACIÓN}

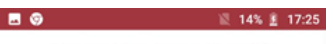

Bienvenida a la app More life En éste apartado encontrarás información sobre e tema de violencia que puede ser de utilidad. familiares y a los contactos que registraste desde el liginin, con la finalidad de que eetén enterados de
ésta app y su forma de proceder. ¿Cómo proceder ante una agresión?

¿Sabías que la violencia tiene diversas tipificaciones?, algunas de ellas son:

Las tipificaciones de la violencia pueden suscitarse en diversos ámbitos:

Directorio de Instituciones de Apoyo. La subsiguiente información fue obtenida de elversos medos entre los que se encuentran proporcionan los contactos de las instituciones
encargadas de asistir a las mujeres en las diversas $\triangleleft$
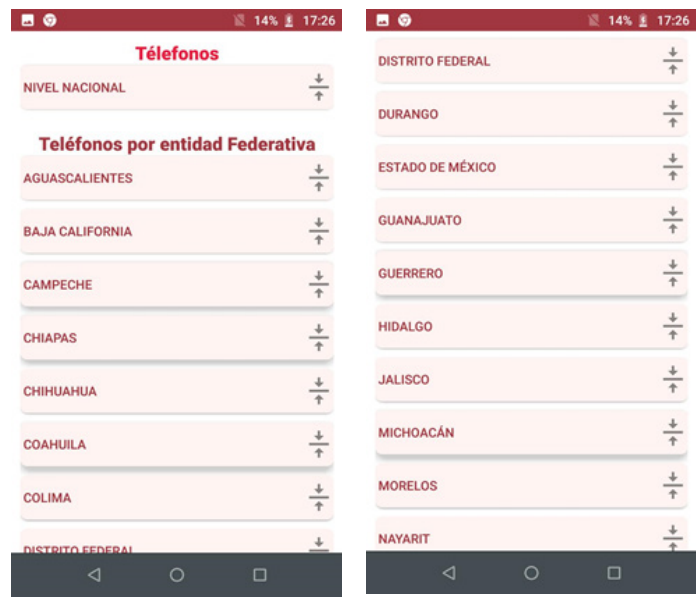
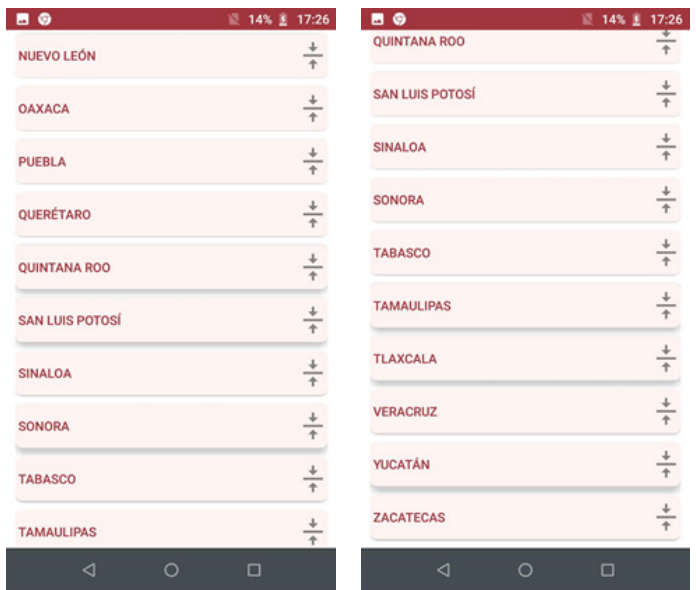

PANTALLAS DE MENSAJES

\section{एक्षित}

\section{MORE LIFE}

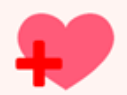

1) enviarmensajes

2

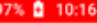

[S $\rightarrow$

\section{CONCLUSIÓN}

La violencia contra las mujeres y las niñas es una de las violaciones de los derechos humanos más graves, extendidas, arraigadas y toleradas en el mundo de acuerdo a la Organización de las Naciones Unidas Mujeres ONU MUJERES, (2018, pág. 1). Las mujeres y las niñas sufren diversos tipos de violencia en todos los ámbitos de su vida y bajo múltiples manifestaciones: en el hogar, en el espacio público, en la escuela, en el trabajo, en el ciberespacio, en la comunidad, en la políitica, en las instituciones, entre otros. El uso de las tecnologías en este tipo de problemáticas ayuda a crear conciencia entre la comunidad además de brindar apoyo a las mujeres víctimas de estas agresiones. 


\section{REFERENCIAS}

Larman C. y Basilli V. (2003). Iterative and Incremental Development: A Brief History. Califonia: IEEE.

EL PAIS. (2017). Android ya es el sistema operativo más usado del mundo. Recuperado de:

https://elpais.com/tecnologia/2017/04/04/actualidad/1491296467_396232.html

ENDIREH. (2016) Encuesta Nacional sobre la Dinámica de las Relaciones en los Hogares 2016. Recuperado de:

http://www.beta.inegi.org.mx/contenidos/programas/endireh/2016/doc/endireh2016_guia_autocuidado.pdf

Instituto Nacional de Estadistica y Geografía. (2017). Fuentes y datos sobre la violencia contra las mujeres en México. Recuperado de: https://www.inegi.org.mx/rde/rde_20/doctos/rde_20_artl.pdf

inMujeres. (2009). Estadística de violencia contra las mujeres en México. Recuperado de: http://estadistica.inmujeres.gob.mx/formas/ convenciones/Nota\%20violencia.pdf

ONU MUJERES. (2018). Día Internacional para la Eliminación de la Violencia contra la Mujer. Recuperado de:

http://mexico.unwomen.org/es/noticias-y-eventos/articulos/2018/11/violencia-contra-las-mujeres 
